\title{
Comparative Performance Evaluation of Three Image Compression Algorithms
}

\author{
Jide Julius Popoola* and Michael Elijah Adekanye \\ Department of Electrical and Electronics Engineering, School of Engineering and \\ Engineering Technology, Federal University of Technology, Akure, Nigeria
}

\begin{abstract}
The advent of computer and internet has brought about massive change to the ways images are being managed. This revolution has resulted in changes in image processing and management as well as the huge space requirement for images' uploading, downloading, transferring and storing nowadays. In guiding against this huge space requirement, images need to be compressed before either storing or transmitting. Several algorithms or techniques on image compression had been developed in literature. In this study, three of these image compression algorithms were developed using MATLAB codes. The three algorithms developed are discrete cosine transform (DCT), discrete wavelet transform (DWT) and set partitioning in hierarchical tree (SPIHT). In order to ascertain which of them is most appropriate for image storing and transmission, comparative performance evaluations were conducted on the three developed algorithms using five performance indices. The results of the comparative performance evaluations show that the three algorithms are effective in image compression but with different efficiency rates. In addition, the comparative performance evaluations results show that DWT has the highest compression ratio and distortion level while the corresponding values for SPIHT is the lowest with those of DCT fall in-between. Also, the results of the study show that the lower the mean square error and the higher the peak signal-to-noise-ratio, the lower the distortion level in the compressed image.
\end{abstract}

Keywords: Image compression, image, image compression methods, performance indices.

\section{Introduction}

Uncompressed multimedia data such as images, graphics, audio and video require considerable large storage capacity and transmission bandwidth. In order to overcome these challenges, these types of data need to be compressed in order to reduce the required storage size as well as the required transferring time and bandwidth. This makes data compression especially image compression an essential process in multimedia computer services and other telecommunication services and applications. This is because image compression, according to [1,2] has inherent capability of reducing the redundancies and irrelevancies present in an image so that the image can be stored and transmitted in a compact and efficient form. Thus, image compression can be defined as the process of removing redundant or unnecessary information from an image so that only the essential information can be retained. It can also be defined, according to [3,4], as a way of minimizing the size in bytes of an image files without degrading the quality of the image to an unacceptable level. Therefore, the

\footnotetext{
* Corresponding author. Tel.: +234-803-4131860; E-mail address: jidejulius2001 @ gmail.com

Manuscript History:

Received 28 December, 2016, Revised 24 March, 2017, Accepted 28 March, 2017, Published 28 April, 2017

ISSN: $2289-7771$

Copyright $\odot 2017$ JASPE

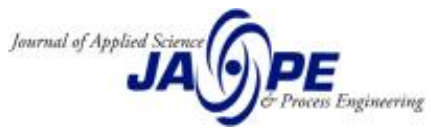


primary aim of image compression is to represent an image file in fewest numbers of bits without losing the important or essential information content in the original image. Obviously, there are two major reasons behind image compression. While the first reason is to enable more images to be stored in an available memory space, the second reason is to reduce both the transmission duration and bandwidth that is required for an image to be either uploaded to or download from the Internet.

There are various applications that require image compression in order to effectively increase both their efficiencies and performances. A typical example of such applications is e-health system that involves transmission of electrocardiogram (ECG) data. Other examples are technical drawing, clip art, natural images such as photographs. Basically, in image compression, the compression degree required as well as the application involved usually determines the appropriate image compression method or technique to be employed. Generally, there are two major types of image compression techniques or methods: lossless and lossy [4-9]. In lossless image compression, the original image can be perfectly reconstructed from the compressed or encoded image [10]. This technique is also referred to as noiseless because it does not add noise to the image. In addition, the technique is also being referred to as entropy code because it uses statistics or decomposition technique to minimize or eliminate redundancy [10]. As a result of its inherent capability, this image compression technique is usually used to compress image in quality-critical applications since it allows the exact original image to be reconstructed from the compressed one without any loss of the image data. Although lossless image compression technique gives good compression quality, its major disadvantage according to [5] is that its compression ratio is usually low. The main application of lossless image compression technique is medical imagery $[10,11]$.

On the other hand, lossy image compression as the name implies allows loss in the actual image such that the original uncompressed image cannot be created exactly from the compressed file. Hence, the reconstructed image in this technique usually has degradation relative to the original image. By this, lossy image compression technique suffers loss of some data during the compression processing. However, the distortion level of the reconstructed image is usually acceptable since it is unnoticeable by human eyes [6]. This implies that the decompressed images of this compression method are not perfectly identical to the original images but they are usually reasonably close [12]. The method thus works by recognizing unnecessary or less relevant information and eliminating it.

Generally speaking, as stated in [9], there are parts of images that are more important than others. Hence, the region(s) of interest in an image is/are usually small when compared to the whole image. Thus, if lossless compression technique is employed in compressing the whole image, the compression ratio obtained is low, which implies that sizable storage and transmission bandwidth are difficult to achieve using lossless compression method. However, since the primary objective of this study is to find a means of reducing the storage capacity and transmission bandwidth required for image storage and transmission, lossy compression technique that has high compression ratio is the focus of this study. Hence, three different lossy compression schemes (DCT, DWT and SPIHT) were developed with improved compression ratio and low computational complexity. The developed compression schemes were evaluated using five performance indices to determine which of the three algorithms gives better image compression quality with minimal distortion level. The performance indices employed are peak-signal-to-noise-ratio (PSNR), mean square error (MSE), compression ratio $(\mathrm{CR})$, algorithm operating time and image histogram. The analyses carried out were based on the amount of distortion and the compression ratio. Details on these performance indices and the compression techniques employed in carrying out the study were presented in this paper.

In ensuring chronological and coherent presentation of the paper, the rest of this paper is organized as follows: In Section 2, brief backgrounds on image and image compression techniques were carried out. In addition, procedural review on each of the three algorithms was also carried out 
and presented in the section. Furthermore, brief review on the five performance indices employed was presented in the section. The methodology involved in carrying out the study is presented in Section 3. The results obtained in Section 3 were analyzed and discussed in Section 4. Section 5, which is the last section, concludes the paper.

\section{Background on image and image compression}

An image, according to [13], is defined as an artifact that shows or records visual perception. Image is a kind of redundant data that contains the same information from certain perspective of view. Hence, by using compression methods, it is possible to remove some of the redundant information contained in images [13]. Irrespective of the image compression method employ, compression method that will guarantee high compression ratio with better accuracy usually involves the basic steps shown in Figure 1. Hence, for a typical lossy image compression method, the first step involved is a mathematical transformation that allows switching from spatial domain to a transform domain where coefficients are low correlated. This step is followed by quantization step while the final step is coding, which produces the stream representing the compressed image.

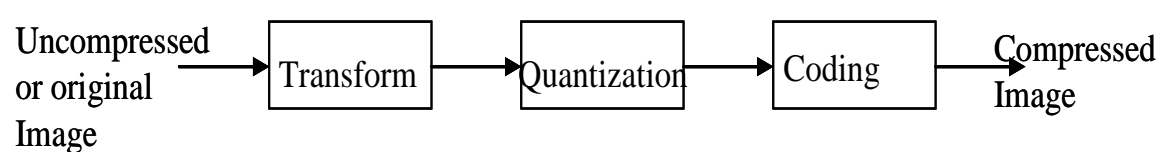

Figure 1. Typical image compression model.

Generally, two major transforms are usually used in image compression. These two transforms are discrete cosine transform (DCT) and discrete wavelet transform (DWT). The two transforms use the principle of de-correlation in the first step of Figure 1 to extract relevant information from the image and reduce redundancy in the image. Basically, the method of image compression is not new as discovery of DCT in 1974 motivated study on image compression. Until recently, the DCT as reported in [14] has been the most popular transform technique for compression as a result of its optimal performance and ability to be implemented at a reasonable cost. Some of the popular emerged standards that employed DCT algorithm or principle in image compression are the Joint Photographic Experts Group (JPEG) [15], Moving Picture Expert Group (MPEG) [16] and Px64 [17]. While JPEG is being used for compression of photograph and still images, MPEG and Px64 are used for compression of motion video, and video telephony and teleconferencing respectively.

However, as reported in [18], a fundamental shift in image compression scheme came after the DWT became popular. This popularity as well as DWT inherit multi-resolution nature aids its widespread acceptance in signal processing and image compression. On the other hand, in 1996, according to [5], Said and Pearlman developed wavelet based image compression algorithm known as set partitioning in hierarchical tree (SPIHT). The SPIHT uses the principle of zero-tree coding from embedded zerotree wavelet (EZW) as well as wavelet decomposition and imposes a quad tree structure across the sub-bands in order to exploit the inter-band correlation. The algorithm uses a special data structure called spatial orientation trees (SOT) instead of an arithmetic encoder with better and efficient compression performance. Brief review on each of these three image compression (DCT, DWT and SPIHT) algorithms employed in this study and their procedural steps are presented in the following subsections. 


\subsection{Discrete cosine transform algorithm}

Basically, there are two types of discrete cosine transform (DCT), namely one dimensional DCT and two dimensional DCT. While the one dimensional DCT is used for processing one-dimensional signals such as speech waveform, the two-dimensional (2D) DCT is used for analyzing signals such as images. Irrespective of the DCT type use, the DCT compression algorithm or technique works by transforming image from spatial domain to the frequency domain. It does the image transformation process by separating the image into spectral sub-bands of differing importance in respect to the image's visual quality. This is achieved by representing the uncompressed or original image points in the form of sum of cosine functions that are oscillating at different frequencies and magnitudes.

Hence, in this study, which is on image compression, the 2D DCT for an NxN input was employed. The 2D DCT employed is defined mathematically in [19] as;

$$
D_{D C T}(i, j)=\frac{1}{\sqrt{2 N}} B(i) B(j) \sum_{x=0}^{N-1} \sum_{y=0}^{N-1} M(x, y) \cdot \cos \left[\frac{2 x+1}{2 N} i \pi\right] \cos \left[\frac{2 y+1}{2 N} j \pi\right]
$$

where $M(x, y)$ is the input image of size $x \times y$,

$$
B(i)=\left\{\begin{array}{cl}
\frac{1}{\sqrt{2}}, & \text { if } i=0 \\
1, & \text { if } i>0
\end{array} \text { and } B(j)=\left\{\begin{array}{cc}
\frac{1}{\sqrt{2}}, & \text { if } j=0 \\
1, & \text { if } j>0
\end{array}\right. \text {. }\right.
$$

In this study, the input image is first divided into $8 \times 8$ blocks and the 8 -point $2 \mathrm{D}$ DCT is performed. The DCT coefficients, according to [19], are then quantized using $8 \times 8$ quantization. The quantization is attained by dividing each elements of the transformed input image matrix by corresponding element in the quantization matrix $\mathrm{Q}$ and rounding to the nearest integer value using the mathematical expression given in [19] as;

$$
D_{\text {quant }}(i, j)=\operatorname{round}\left\{\frac{D_{D C T}(i, j)}{Q(i, j)}\right\}
$$

where $Q(i, j)=\left[\begin{array}{ccccc}q_{11} & q_{12} & \cdots & q_{1(j-1)} & q_{1 j} \\ q_{21} & q_{22} & \cdots & q_{2(j-1)} & q_{2 j} \\ \vdots & \vdots & \vdots & \vdots & \vdots \\ q_{(i-1) 1} & q_{(i-1) 2} & \cdots & q_{(i-1)(j-1)} & q_{(i-1) j} \\ q_{i 1} & q_{i 2} & \cdots & q_{i(j-i)} & q_{i j}\end{array}\right]$

During quantization stage as shown in Figure 1, the less essential frequencies are discarded while the essential frequencies that remain are used in retrieving the image during decomposition stage. After the quantization, some of the higher frequency coefficients will become zero as a result of DCT energy compaction attribute. The compression is achieved by employing appropriate scaling 
factor. The quantized coefficients are then rearranged in a zigzag order, as reported in [20], to further compress the image by an efficient lossy coding algorithm.

The four basic activities or procedures involved in DCT image compression is summarized as follows: Firstly, the uncompressed image to be compressed is broken into $N \times N$ blocks, where $N=2^{n} ; \quad n=2,3,4$, etc. Secondly, the DCT is applied to each block working from left to right and top to bottom. Thirdly, each block's element is compressed through quantization means. Lastly, the array of compressed blocks that constitute image is stored in a drastically reduced amount of space.

\subsection{Discrete wavelet transform algorithm}

In discrete wavelet transform (DWT), an uncompressed image is represented by sum of wavelet functions, which are known as wavelets. These wavelets have different locations and scales. Basically DWT represents the image to be compressed into a set of high pass coefficients called details and low pass coefficients also known as approximates. The uncompressed or original image is first divided into $N \times N$ blocks, where $N=32$. Each block is then passed through two filters [19]. In the first filter, decomposition is performed to decompose the input image into detail and approximate coefficients. The obtained detail and approximate coefficients are then separated into $\mathrm{LL}_{\mathrm{n}}, \mathrm{HL}_{\mathrm{n}}, \mathrm{LH}_{\mathrm{n}}$ and $\mathrm{HH}_{\mathrm{n}}$ coefficients where $\mathrm{LL}_{\mathrm{n}}$ is low pass filter over rows and columns, $\mathrm{HL}_{\mathrm{n}}$ is high pass filter over rows and low pass filter over columns, $\mathrm{LH}_{\mathrm{n}}$ is low pass filter over rows and high pass filter over columns and $\mathrm{HH}_{\mathrm{n}}$ is high pass filter over rows and over columns. The result, according to [21], is a four sub-band term $\mathrm{LL}_{1}$ (horizontally and vertically low pass), $\mathrm{HL}_{1}$ (horizontally high pass and vertically low pass), $\mathrm{LH}_{1}$ (horizontally low pass and vertically high pass) and $\mathrm{HH}_{1}$ (horizontally and vertically high pass). In order to increase the efficiency of the DWT, multiple decomposition stage is recursively performed on the $L_{1}$ sub-band to smoothed version of the original image. Thus $L_{2}$ is decomposed to $\mathrm{LL}_{2}, \mathrm{HL}_{2}, \mathrm{LH}_{2}$ and $\mathrm{HH}_{2}$ as shown in Figure 2.

\begin{tabular}{|c|l|l|l|}
\hline $\mathrm{LL}_{3}$ & $\mathrm{HL}_{3}$ & \multirow{2}{*}{$\mathrm{HL2}$} & \\
\cline { 1 - 2 } $\mathrm{LH}_{3}$ & $\mathrm{HH}_{3}$ & \\
\cline { 1 - 2 } $\mathrm{LH}_{2}$ & $\mathrm{HH}_{2}$ & \\
\hline & & \multirow{2}{*}{$\mathrm{HH}_{2}$} \\
\multicolumn{2}{|c|}{$\mathrm{LH}_{1}$} & & \\
& & \\
& & \\
\hline
\end{tabular}

Figure 2. An image of 3-level 2D DWT decomposition [21].

Generally, the basic procedures involved in DWT image compression are summarized as follows: Firstly, a wavelet is chosen. Then choose decomposition level $\mathrm{N}$ and compute the wavelet. Secondly, for each level from 1 to $\mathrm{N}$, a threshold is decided and applied to the detail coefficients. Finally, wavelet reconstruction is computed using the original approximation coefficients of level $\mathrm{N}$ and the modified detail coefficients of levels from 1 to $\mathrm{N}$. 


\subsection{Set partitioning in hierarchical tree algorithm}

The set partitioning in hierarchical tree (SPIHT), according to [5], uses wavelet sub-band decomposition and imposes a quad tree structure across the sub-bands in order to exploit the interband correlation. The algorithm according to [5], searches each tree, and partitions the tree into one of these three lists: one, the list of significant pixels (LSP) containing the coordinates of pixels found to be significant at the current threshold; two, the list of insignificant pixels (LIP), with pixels that are not significant at the current threshold; and three, the list of insignificant sets (LIS), which contain information about trees that have all the constituent entries to be insignificant at the current threshold. Figure 3 shows the tree structure for level 2 decomposition in SPIHT algorithm.

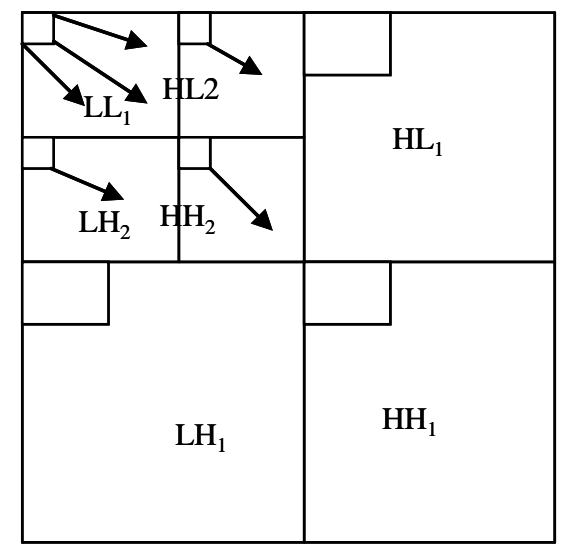

Figure 3. Tree structure used in SPIHT algorithm [5].

Basically, the procedures involved in SPIHT image compression algorithm is divided into three stages, namely: initialization, sorting pass and refinement pass. The activities involved in each stage are summarized as follows: Firstly, at the initialization stage, a start threshold is defined by the algorithm according to the maximum value in the wavelet coefficients pyramid and sets the LSP as an empty list and puts the coordinates of all coefficients in the coarsest level of the wavelet pyramid ( $L L$ band) in the LIP and those which have descendants to the LIS. Secondly, at the sorting pass, features in the LIP and the LIS are sorted. Significance test is carried out on each pixel in LIP matched against the current threshold and outputs the test result ( 0 or 1$)$ to the output bit-stream. For significant coefficient, its coordinate is moved to the LSP. The sorting pass of LIS, SPIHT does the significant test for each set in the LIS and outputs the significance information (0 or 1). If a set is significant, it is partitioned into its offspring and leaves. The current threshold is divided by 2 . Finally, according to [22], the sorting and refinement stages are continued until target bit-rate is achieved.

\section{Methodology}

In carrying out this study, the uncompressed or original images used for the study were acquired using smart-camera. The acquired images were then stored on secured digital or memory card of the camera and consequently transferred to the memory of the computer used. This is to enhance easy accessibility to the images as well as to allow further processing on the images such as extraction of 
some features from the acquired images. The information extracted includes the image size, format, color depth, alignment and color. This extraction process serves as a prelude to the compression process. MATLAB function was written to get the details of the images. Also, MATLAB functions were developed for the three image compression algorithms reviewed in last section. The image compression process was then carried out in MATLAB environment. The acquired uncompressed images were compressed using each of the three algorithms developed. After the successful development of the three image compression algorithms, the performance of each of them was evaluated.

In carrying out the performance evaluation of the three algorithms, five performance indices were employed. The five performance indices employed as mentioned earlier are peak-signal-tonoise-ratio (PSNR), mean square error (MSE), compression ratio (CR), algorithm operating time and image histogram. PSNR is a measure of peak error in compressed image. It is the most commonly used performance index to measure the quality of reconstruction of lossy image compression. It is evaluated in decibel using the mathematical expression defined in [19] as;

$$
P S N R=10 \log _{10} \frac{I^{2}}{M S E}
$$

where $I$ is a constant known as allowable image pixel intensity level. On the other hand, MSE is the cumulative squared error between the compressed image and the original or uncompressed image. It is defined mathematically in $[23,24]$ as;

$$
M S E=\frac{1}{M N} \sum_{x=1}^{M} \sum_{y=1}^{N}[f(x, y)-\hat{f}(x, y)]^{2}
$$

where $f(x, y)$ is the original image, $\hat{f}(x, y)$ is the compressed image, $M$ and $N$ are the matrix dimensions in $x$ and $y$ respectively. Basically, PSNR and MSE are the two error matrices usually used to compare compression quality. Technically, the higher the value of PSNR, the better the quality of the obtained compressed image.

Compression ratio (CR), on the other hand, is an engineering term used to quantify the reduction in image size produced by image compression algorithm. It is the ratio of the original image size to that of the compressed image. It is defined in [24] as the nominal bit depth of the original image in bits per pixel divided by the bits per pixel necessary to store the compressed image. It is expressed mathematically in [23] as;

$$
C R=\frac{n_{1}}{n_{2}}
$$

where $n_{1}$ and $n_{2}$ are the original size or number of bits in original image and the compressed image respectively. Thus, a CR of 5 indicates that the original image's size has 5 bits for every 1 unit in the compressed image. In addition, the image histogram is another performance index used in this study. Histogram of an image, like other histogram, shows frequency. However, an image histogram shows frequency of pixels intensity values that portrays the gray level intensities on horizontal axis and the frequency of these intensities on the vertical axis. The last performance metric index employed is the 
algorithm operating time. This gives the time taken for the each algorithm to compress a specific image. The results obtained when the three developed image compressed algorithms were evaluated using the five performance indices are presented and discussed in next section.

\section{Experimental Results and Discussion}

After the successful development of the three image-compression algorithms, their respective efficiency in image compression capability were evaluated using the five performance indices described above. The three original images used in evaluating the performance of the three developed image compression algorithms are shown in Figure 4. The results obtained in each case are presented and discussed in the following subsections.
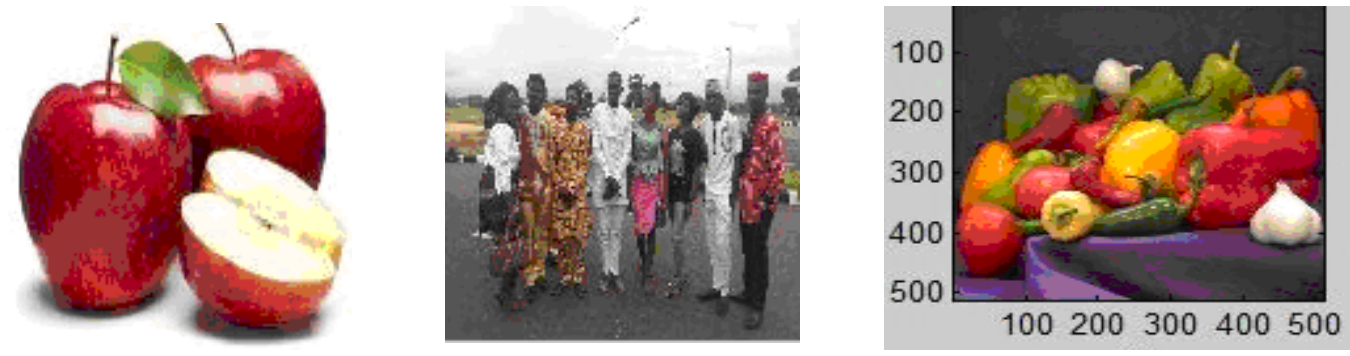

Figure 4. Original images for the performance evaluation analysis.

\subsection{The algorithms performance evaluation using image histogram}

The first performance index employed in evaluating the effectiveness of the three developed algorithms is image histogram. In line with the primary objective of this study, the performance index was to determine reduction in original image size with minimal distortion. In this regard, critical observation of the plotted image histograms in Figures $5-7$ show that DWT, as shown in Figures 5(ii)(d), 6(ii)(d) and 7(ii)(d), relatively outperforms both DCT and SPIHT with SPIHT algorithm, as shown in Figures 5(iii)(d), 6(iii)(d) and 7(iii)(d), directly follows that of DWT while that of DCT algorithm, as shown in Figures 5(i)(d), 6(i)(d) and 7(i)(d), is the lowest. This is clearly buttressed in the Figure 5(ii)(b), Figure 6(ii)(b) and Figure 7(ii)(b) respectively. However, since minimal distortion in image quality as well as reduction in size is to be considered, SPIHT algorithm is therefore recommended as the most effective of the three algorithms based on the compressed image direct resemblance and comparative reduction in original image size. 


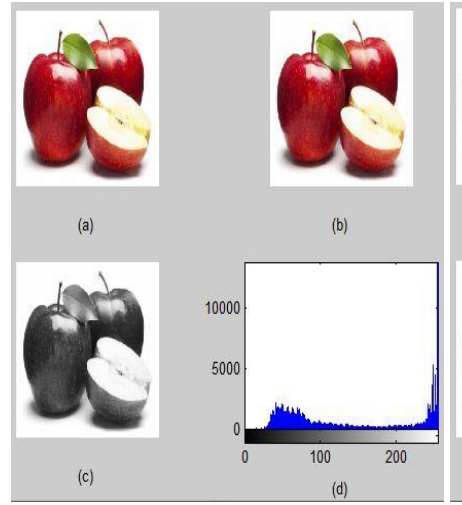

(i)

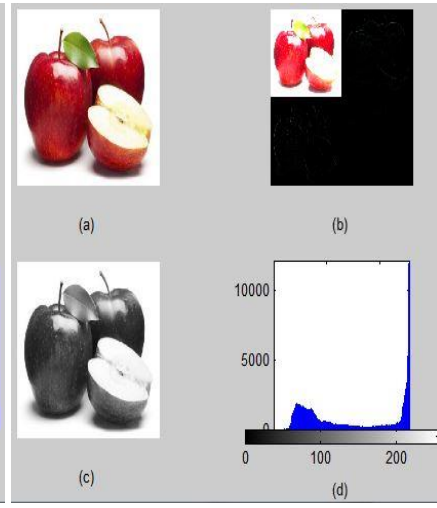

(ii)

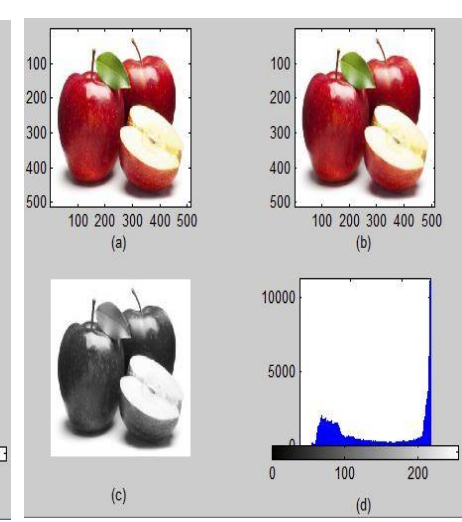

(iii)

Figure 5. (i) DCT, (ii) DWT and (iii) SPIHT performance evaluations results for apple.

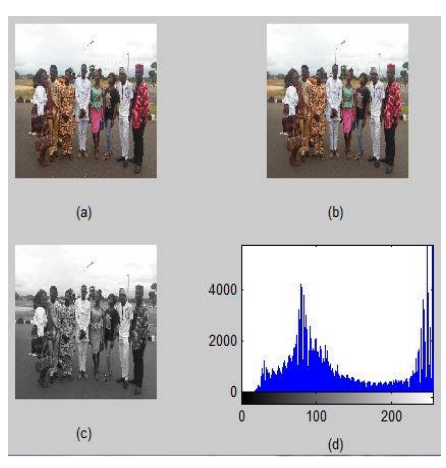

(i)

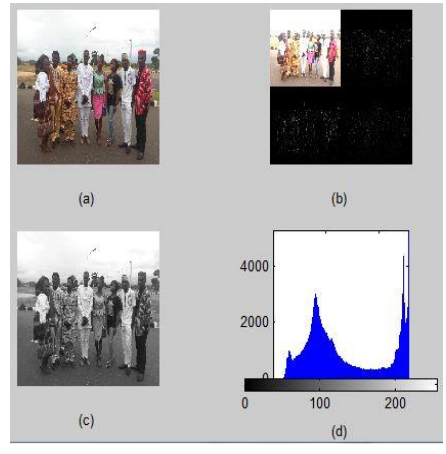

(ii)

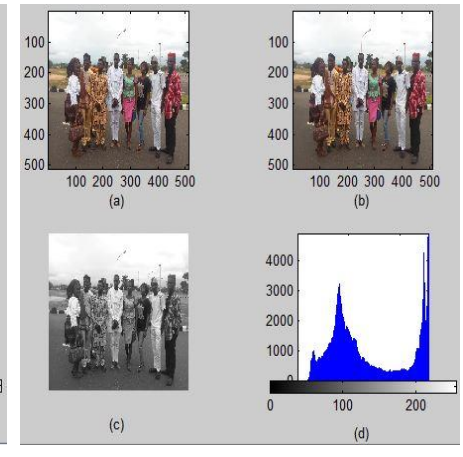

(iii)

Figure 6. (i) DCT, (ii) DWT and (iii) SPIHT performance evaluations results for picture.

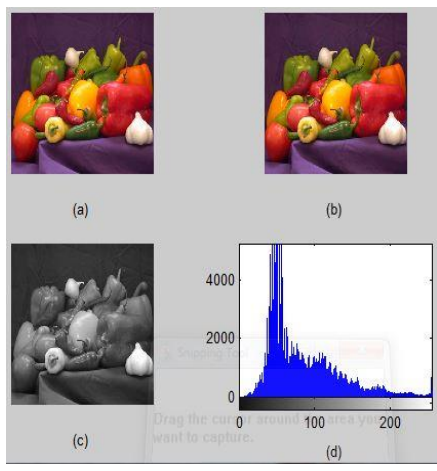

(i)

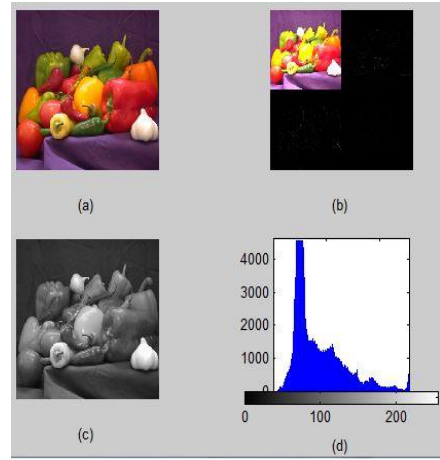

(ii)

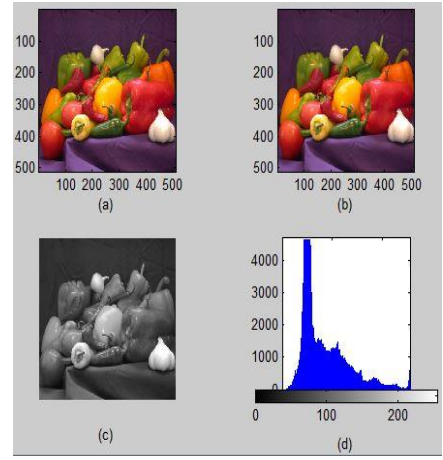

(iii)

Figure 7. (i) DCT, (ii) DWT and (iii) SPIHT performance evaluations results for pepper. 


\subsection{The algorithms performance evaluation using compression ratio (CR)}

$\mathrm{CR}$ as defined above is a compression performance index that quantifies the reduction in image size produced by image compression algorithm. Hence, the higher the CR value obtained from an image compressing algorithm, the better the algorithm that produces it. Thus, as shown in Figure 8, DWT algorithm outperforms the two other algorithms while SPIHT directly follows DWT and DCT is the last for the three images used in evaluating the performance of the three developed image compression algorithm.

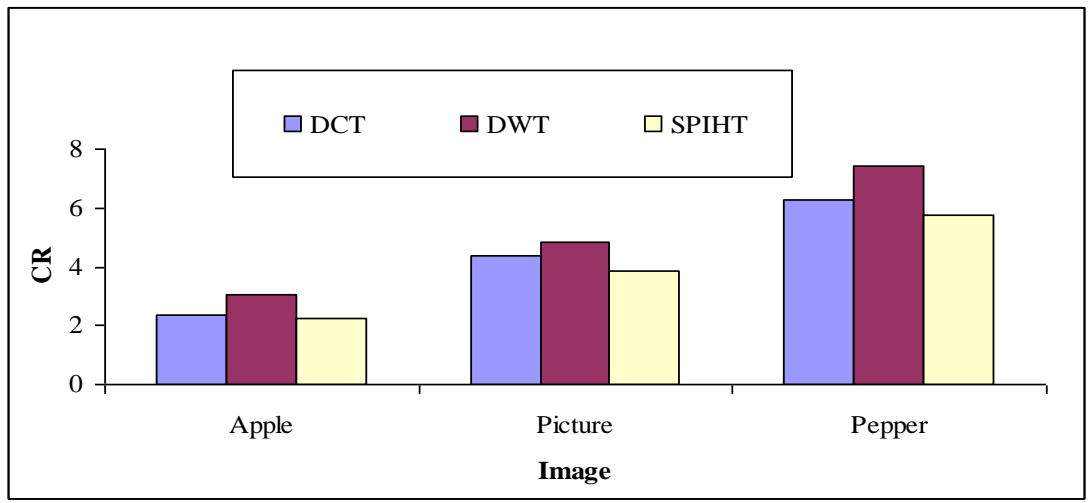

Figure 8. CR comparative performance results for the three algorithms.

\subsection{The algorithms performance evaluation using PSNR and MSE}

Furthermore, in assessing the comparative performance evaluations of the three developed image compression algorithms for this study, MSE and PSNR, which are two of the most popular performance indices to estimate the efficiency and effectiveness of image compressing algorithm, were employed using the three original images as test samples. The experimental results obtained for MSE and PSNR are presented graphically in Figures 9 and Figure 10 respectively. From the results presented in Figure 9 and Figure 10 as well as equation (4), it is obvious that there is inverse proportionality relationship between MSE and PSNR. Hence, from the observations of the experimental results of PSNR and MSE, it is deduced that when assessing the reconstructed images under these performance indices, SPIHT showed good performance throughout with better quality of images at the same time. Therefore, it can be established that SPIHT is the best algorithm based on the experimental results of this study. This inference is justified on MSE result in Figure 9 as errors introduced in those images by DWT and DCT algorithms for each image is relatively higher than the corresponding error introduced by SPIHT algorithm. Therefore, since the higher the MSE value the higher the distortional value introduced, it is obvious that only SPIHT algorithm reproduced the sample images with minimal distortion level. 


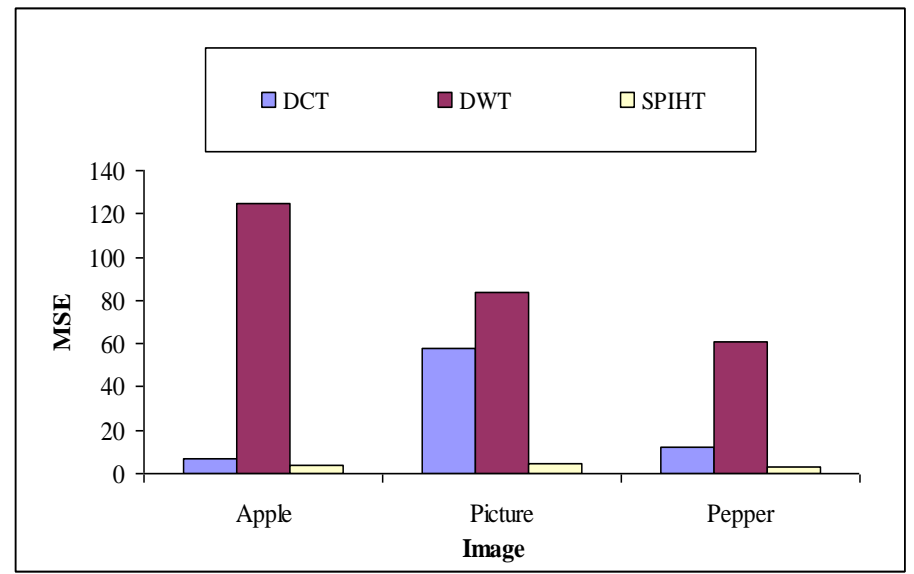

Figure 9. MSE comparative performance results for the three algorithms.

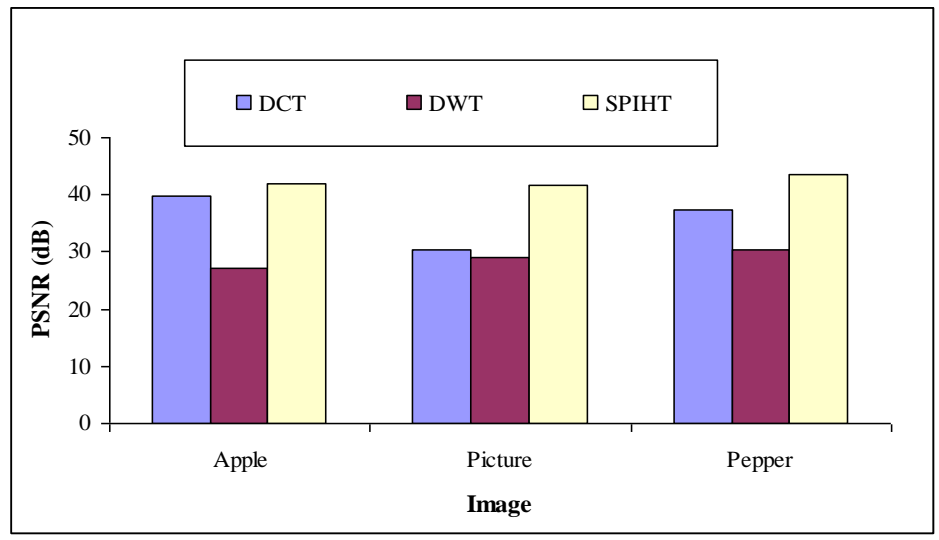

Figure 10. PSNR comparative performance results for the three algorithms.

\subsection{The algorithms performance evaluation using operating time and compression distortion}

Critical observations of Table 1 show that DWT algorithm has least computational complex. On the other hand, DCT and SPIHT algorithms are relatively more computational complex than DWT algorithm. This accounts for the reason why the operating time for SPIHT is the highest for the same input image while that of the DWT is the lowest with that of DCT in between them. In addition, Table 1 shows that for the same input image DWT algorithm has the least percentage level of image compression among the three algorithms. Furthermore, critical observations of Figure 9 and Table 1 show that DWT algorithm gives considerably highest distortion in image compression size follows by DCT algorithm while SPIHT algorithm gives the lowest. Hence, with the this highest level of distortion by DWT algorithm as shown in Figure 9 and buttresses numerically in Table 1, SPIHT is found to be the best among the three algorithms because of its comparably better image compression quality and minimal distortion level advantage while DWT algorithm is the least and DCT algorithm is between the two extremists for the same reason. 
Table 1. Developed algorithms comparative analysis in terms of complexity and distortion

\begin{tabular}{|c|c|c|c|c|c|c|c|}
\hline Algorithm & $\begin{array}{c}\text { Input } \\
\text { Image }\end{array}$ & $\begin{array}{c}\text { Operating } \\
\text { Time (s) }\end{array}$ & $\begin{array}{c}\text { Size of } \\
\text { Input } \\
\text { Image } \\
\text { (byte) }\end{array}$ & $\begin{array}{c}\text { Size of } \\
\text { Compressed } \\
\text { Image (byte) }\end{array}$ & $\begin{array}{c}\text { Level of } \\
\text { Image } \\
\text { Compression } \\
(\boldsymbol{\%})\end{array}$ & $\begin{array}{c}\text { Compressed } \\
\text { Image } \\
\text { Distortion }\end{array}$ & $\begin{array}{c}\text { Level of } \\
\text { Image } \\
\text { Distortion } \\
(\%)\end{array}$ \\
\hline \multirow{3}{*}{ DCT } & Apple & 9.1263 & 49352 & 20944 & 42.4 & 28408 & 57.6 \\
\cline { 2 - 8 } & Picture & 10.1290 & 162664 & 37035 & 22.8 & 125629 & 77.2 \\
\cline { 2 - 8 } & Pepper & 9.8561 & 153100 & 24438 & 16.0 & 128662 & 84.0 \\
\hline \multirow{3}{*}{ DWT } & Apple & 1.3972 & 49352 & 16176 & 32.8 & 33176 & 67.2 \\
\cline { 2 - 8 } & Picture & 1.4291 & 162664 & 33808 & 20.8 & 128856 & 79.2 \\
\cline { 2 - 8 } & Pepper & 1.4706 & 153100 & 20581 & 13.4 & 132519 & 85.6 \\
\hline \multirow{3}{*}{ SPIHT } & Apple & 11.9913 & 49352 & 21841 & 44.3 & 27511 & 55.7 \\
\cline { 2 - 8 } & Picture & 25.8389 & 162664 & 41932 & 25.8 & 120732 & 74.2 \\
\cline { 2 - 8 } & Pepper & 15.2841 & 153100 & 26709 & 17.4 & 126391 & 82.6 \\
\hline
\end{tabular}

\section{Conclusion}

This study presents three developed image compression algorithms. The performance evaluations of the three developed algorithms were determined using five performance indices. The results of the performance evaluations conducted show that the three developed algorithms performed favorably well with varying compression ratios and different qualities of images. Also, the results of the performance evaluations of the three developed algorithms show that the three algorithms are effective in image compression capability though with different efficiency values. Furthermore, the experimental results of the computational distortional analysis conducted on the three developed image compression algorithms show that SPIHT algorithm has minimal distortion level followed by DCT algorithm, while DWT algorithm has the highest distortional percentage value. Hence, in terms of image size reduction, DWT algorithm is the best among the three algorithms followed by DCT algorithm while SPIHT algorithm is the last. However, since the objective of this study is to determine the best algorithm with minimal distortional value among the three algorithms, it therefore concludes base on the obtained results that SPIHT algorithm is the best followed by DCT while DWT algorithm is the last.

In addition, the results from this study have clearly shown the weaknesses and strengths of each of the three algorithms considered, which most of the related studies in the surveyed literature were not considered. Another contribution of this study to knowledge is in its development of three different image compression algorithms, which is rare in other related studies as most of related studies if not all were based on two algorithms. Finally, based on the results from this study, it is recommended that for application that requests only image size reduction without considering distortion level, DWT can be employed. On the other hand, when distortion level is required, SPIHT algorithm is the best to be applied among the three algorithms considered based on its lowest image distortion level capability. 


\section{References}

[1] Singh, Singh, A.K. and Tripathi, G.S. (2014). A Comparative Study of DCT, DWR and hybrid (DCTDWT) Transform, GJESER Review Paper, Vol. 1, No. 4, pp. 16-21.

[2] Dhawan, S. (2011). A Review of Image Compression and Comparison of its Algorithms, International Journal of Electronics and Communication Technology, Vol. 2, No. 1, pp. 22-26.

[3] Gupta, B. (2013). Study of Various Lossless Image Compression Techniques, International Journal of Emergimg Trends and Technology in Computer Science, Vol. 2, No. 4, pp. 253-257.

[4] Rehman, M., Sharif, M. and Raza, M. (2014). Image Compression: A Survey, Research Journal of Applied Sciences, Engineering and Technology, Vol. 7, No. 4, pp. 256-672.

[5] Nivedita, M. Singh, P., and Jindal, S. (2012). A Comparative Study of DCT And DWT-SPIHT. International Journal of Computational Engineering and Management, Vol. 15, No. 2, pp. 26-32.

[6] Pensiri, F. and Auwatanamongkol, S. (2012). A Lossless Image Compression Algorithm Using Predictive Coding Based on Quantized Colors, WSEAS Transactions on Signal Processing, Vol. 8, No. 2, pp. 43-53.

[7] Alarabeyyat, A., Al-Hashemi S., Khdour, T., Btoush, M.H., Bani-Ahmad, S. and Al-Hashemi, R. (2012), Lossless Image Compression Technique Using Combination Methods, Journal of Software Engineering and Applications, Vol. 5, pp. 752-763.

[8] Weinberger, M.J., Seroussi, G. and Sapiro, G. (2000). The LOCO-I Lossless Image Compression Algorithm: Principles and Standardization into JPEG-LS, IEEE Trans. on Image Processing, Vol. 9, No. 8, pp. 1309-1324.

[9] Zuo, Z., Lan, X., Deng, L., Yao, S. and Wang, X. (2015). An Improved Medical Image Compression Technique with Lossless Region of Interest. Optik-International Journal for Light and Electron Optics, Vol. 126, No. 21, pp. 2825-2831.

[10] Tomar, R.R.S. and Jain, K. (2016). Lossless Image Compression Using Differential Pulse Code Modulation and its Application, International Journal of Signal Processing, Image Processing and Pattern Recognition, Vol. 9, No. 1, pp. 197-202.

[11] Masood, S., Sherif, M., Yasmin, M., Raza, M. and Mohsin, S. (2012). Brain Image Compression: A Brief Survey. Research Journal of Applied Sciences, Engineering and Technology, Vol. 5, No. 1, pp. 49-59.

[12] Marimuthu, M., Muthaiah, R. and Swaminathan, P. (2012). Review Article: An Overview of Image Compression Techniques. Research Journal of Applied Sciences, Engineering and Technology, Vol. 4, No. 24, pp. 5381-5386.

[13] Vijayvargiya, G., Silakari, S. and Pandy, R. (2013). A Survey: Various Techniques of Image Compression. International Journal of Computer and Information Security, Vol. 11, No. 10, pp. 51-55.

[14] Rehna, V.J.and Jeya Kumar, M.K. (2012). Wavelet Based Image Coding Schemes: A Recent Survey, International Journal of Soft Computing, Vol. 3, No. 3, pp. 101-118.

[15] Wallace, G.K. (1991). The JPEG Still Picture Compression Standard, Communications of the ACM Magazine, Vol. 34, No. 4, pp. 30-44.

[16] Puri, A. (1992). Video Coding Using the MPEG-1 Compression Standard, Society for Information Display Digest of Technical Papers, Vol. 23, pp. 123-126.

[17] Watson, A.B. (1994). Image Compression Using the Discrete Cosine Transform, Mathematica Journal, Vol. 4, No. 1, pp. 81-88.

[18] Yadavi, R.J., Gangwar, S.P. and Singh, H.V. (2012). Study and Analysis of Wavelet Based Image Compression Techniques International Journal of Engineering, Science and Technology, Vol. 4, No. 1, pp. $1-7$.

[19] Bindu, K., Ganpati, A. and Sharma, A.K. (2012). A Comparative Study of Image Compression Algorithms, International Journal of Research in Computer Science, Vol. 2, No. 5, pp. 37-42.

[20] Deshlahral, A., Shirnewar, G.S. and Sahoo, A.K. (2013). A Comparative Study of DCT, DWT and Hybrid (DCT-DWT) Transform, In Proceedings International Conference on Emerging Trends in Computer and Image Processing, February 24, pp. 1-7. 
[21] Al-Janabi, A.K. (2013). Low Memory Set-Partitioning in Hierarchical Trees Image Compression Algorithm, International Journal of Video and Image Processing and Network Security, Vol. 13, No. 2, pp. 12-18.

[22] Rema, N.R., Binu A.O. and Mythili, P. (2015). Image Compression Using SPIHT with Modified Spatial Orientation Tress, Procedia Computer Science, Vol. 46, pp. 1732-1738.

[23] Chowdhury, M.M.H. and Khatun, A. (2012). Image Compression Using Discrete Wavelet Transform, International Journal of Computer Science Issues, Vol. 9, No. 1, pp. 327-330.

[24] Saffor, A., Ramli, A R. and Ng, K-H. (2001). A Comparative Study of Image Compression between JPEG and WAVELET, Malaysian Journal of Computer Science, Vol. 14, No. 1, pp. 39-45. 